\title{
Verrundete Kanten, sichere Prozesse, weniger Verschleiß
}

\section{Es klingt wie ein Widerspruch: Schneidkanten zuerst auf maximale Kantenschärfe schleifen, um sie anschließend zu verrunden. Doch das Gleitschleifen sorgt für eine optimierte Oberfläche, verbessert die funktionalen Eigenschaften des Werkzeugs und erhöht die Prozesssicherheit.}

Um die Leistungsfähigkeit der produzierten Fräser für die Metallzerspanung zu verbessern, investierte der schwäbische Hersteller Wunschmann knapp 80.000€ in eine Schleppfinish-Anlage von Otec (Typ DF-3). „Eigentlich widerspricht es ja dem gesunden Menschenverstand“, gibt Geschäftsführer Stephan Wunschmann zu, „erst schleifen wir die Schneidkanten unserer Fräswerkzeuge auf maximale Kantenschärfe, um sie anschließend wieder in der Schleppfinish-Anlage zu verrunden.“ Obwohl es wie ein Wiederspruch klingt, ergibt dieses Vorgehen Sinn: Beim Schleppfinish-Verfahren, auch Gleitschleifen genannt, werden die zu bearbeitenden Werkstücke in speziellen Haltevorrichtungen fixiert und mit hoher Geschwindigkeit in kreisförmiger Bewegung durch einen Behälter mit Schleif- oder Poliergranulat geschleppt. Durch die schnelle Bewegung entsteht ein hoher Anpressdruck zwischen

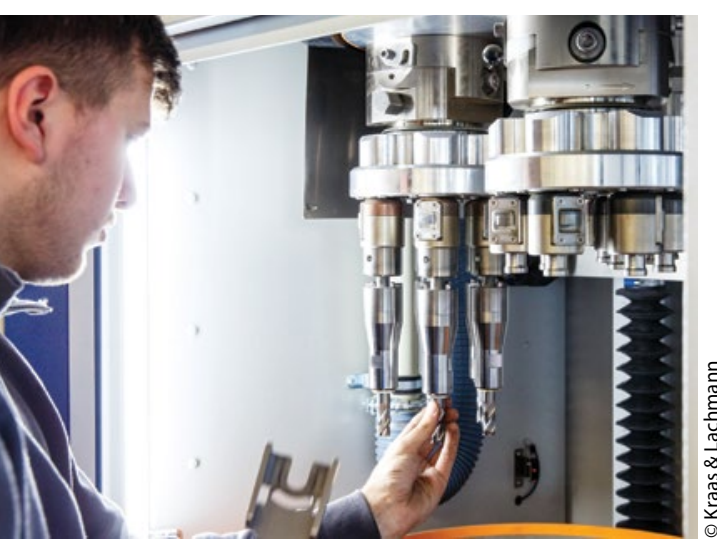

Bei Wunschmann werden die Schneidkanten der Fräswerkzeuge erst auf maximale Kantenschärfe geschliffen und anschließend wieder verrundet.
Werkstück und Verfahrensmittel, der in kürzester Zeit ein optimales Bearbeitungsergebnis in Form exakter Kantenverrundung, Glättung oder eines Hochglanzfinishs in der Qualität einer Handpolitur erzielt.

Zwischen 30 und 60 min dauert der Schleppfinish-Prozess auf der neuen Anlage, die bei Wunschmann in Hailfingen seit ein paar Monaten in Betrieb ist und sich bereits bewährt hat: „Obwohl manche Kunden anfänglich kritisch sind, mich hat das Gleitschleifen überzeugt. Natürlich nicht pauschal für alle Werkzeuge, sondern ganz gezielt und immer mit exakt definierten Werten für die Kantenverrundung“, erklärt der erfahrene Werkzeugspezialist.

\section{Bissig und verschleißfest}

Die Schneidkantenpräparation trägt zum Beispiel bei dem Hochleistungsfräser HPC-Vplus $187 \mathrm{zu}$ einer Steigerung der Standzeit von rund $30 \%$ beim Fräsen von Chrom-Nickel-Stahl (1.4301) bei. „Trotz Verrundung bleibt der Biss des Werkzeugs erhalten, zusätzlich nehmen Verschleißfestigkeit und Prozesssicherheit zu“, sagt Wunschmann, „unbehandelt wären die Schneidkanten bei diesem Fräser schartiger und würden eher zu unkontrolliertem Verschleiß neigen. Verrundete Schneidkanten verschleißen langsamer und gleichmäßiger."

Wunschmann setzt die Gleitschleifanlage aber nicht nur zur Kantenverrundung ein, sondern auch zum Polieren von Spannuten bei Fräswerkzeugen, was wiederum zu mehr Zerspanungsleistung und besserer Spanabfuhr führt. Selbst bei nach-

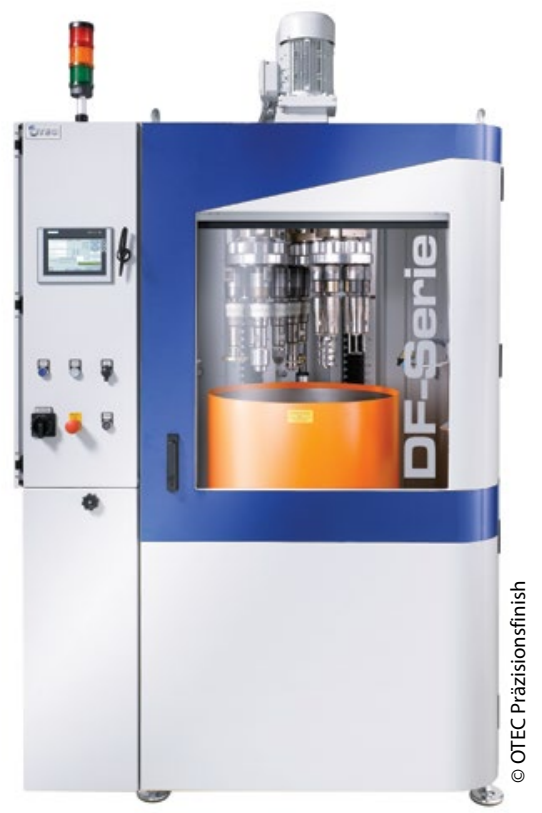

In der Schleppfinish-Anlage werden die zu bearbeitenden Werkstücke mit hoher Geschwindigkeit durch das Schüttgut gezogen.

geschliffenen Werkzeugen können eine Kantenverrundung oder das Polieren von Spannuten zu mehr Leistung führen. //

\section{Kontakte}

OTEC Präzisionsfinish GmbH

Straubenhardt-Conweiler

info@otec.de

www.otec.de

\section{Wunschmann GmbH}

Präzisionswerkzeuge

Rottenburg-Hailfingen www.wunschmann.de info@wunschmann.de 